\title{
Práticas de Integração Ensino-Serviço-Comunidade: a experiência do cuidado à saúde mental na estratégia saúde da família
}

Teaching-Service-Community Integration Practices: the experience of health care mental in the family health strategy

Prácticas de Integración Ensiñanza-Servicio-Comunidad: la experiencia del cuidado de la salud mental em la estratégia de salud de la familia

Bruna Lago Santos

ORCID: https://orcid.org/0000-0002-0733-0585 Universidade Estadual do Sudoeste da Bahia, Brasil E-mail: bhu.lago@hotmail.com

Alana Clara Santana Nascimento

ORCID: https://orcid.org/0000-0003-0656-8951 Universidade Estadual do Sudoeste da Bahia, Brasil E-mail: lanaclaras@hotmail.com

Ana Paula de Souza Cunha

ORCID: https://orcid.org/0000-0002-2567-0647 Universidade Estadual do Sudoeste da Bahia, Brasil E-mail: souzacunhaanapaula@gmail.com

Dornelio Arcanjo de Brito

ORCID: https://orcid.org/0000-0001-6501-7434 Universidade Estadual do Sudoeste da Bahia, Brasil E-mail: dorneliouesb2017@gmail.com

Flávio Gabriel Barbosa Mendes

ORCID: https://orcid.org/0000-0001-8352-0623 Universidade Estadual do Sudoeste da Bahia, Brasil

E-mail: flaviogabrielbarbosa@gmail.com

José Santana Farias Neto

ORCID: https://orcid.org/0000-0002-4073-7838 Universidade Estadual do Sudoeste da Bahia, Brasil E-mail: jsfneto2@gmail.com

Leonardo Victor Costa Reis

ORCID: https://orcid.org/0000-0001-7541-1451 Universidade Estadual do Sudoeste da Bahia, Brasil E-mail: 1victor.reis@gmail.com

Paulo Sérgio Almeida Silva Júnior

ORCID: https://orcid.org/0000-0003-1705-4948 Universidade Estadual do Sudoeste da Bahia, Brasil E-mail: paulasasjunior@gmail.com

Stefanie Sousa de Queiroz

ORCID: https://orcid.org/0000-0002-8549-8340 Universidade Estadual do Sudoeste da Bahia, Brasil E-mail: stefsqueiroz@gmail.com

Thaís Pires de Melo Ximenes

ORCID: https://orcid.org/0000-0003-3405-6531 Universidade Estadual do Sudoeste da Bahia, Brasil E-mail: thaispmx@hotmail.com

\begin{abstract}
Resumo
Objetivo: explanar as vivências de graduandos do segundo ano de medicina, relativas à implantação do projeto de intervenção em saúde mental em uma Unidade de Saúde da Família (USF) no município de Jequié, Bahia, Brasil. Metodologia: trata-se do relato de experiência, evidenciando a construção do projeto de intervenção da disciplina Práticas de Integração Ensino, Serviço e Comunidade e os resultados alcançados na USF. Foram realizados acompanhamentos das atividades: visitas domiciliares, palestras em sala de espera, Práticas Integrativas e Complementares, feira de saúde, e matriciamento; complementando o estudo, foi utilizada a ferramenta SelfReporting Questionnaire (SRQ-20) e a capacitação dos profissionais de saúde para uso posterior da ferramenta na comunidade. Resultados: no contexto dos discentes, o vínculo com a comunidade possibilitou o conhecimento do cotidiano dos pacientes, em seu âmbito biopsicossocial, e contribuiu para a formação médica humanizada. Os
\end{abstract}


profissionais demonstraram interesse na melhoria dos serviços oferecidos, embora houvesse uma resistência na operacionalização das atividades na rotina da unidade. A população que participou das atividades propostas avaliou-as de forma positiva, apesar da baixa adesão, limitando o alcance das intervenções. Conclusão: a experiência vivenciada mostrou-se benéfica para a consolidação de uma nova forma de cuidado à saúde mental dos pacientes da USF, consistindo na integração ensino-serviço-comunidade.

Palavras-chave: Educação em saúde; Educação médica; Atenção primária à saúde; Saúde mental.

\begin{abstract}
Objective: to explain the experiences of second-year medical students regarding the implementation of the intervention project in mental health in a Family Health Unit (FHU) in the city of Jequié, Bahia, Brazil. Methodology: this is an experience report, showing the construction of the intervention project of the discipline Integration Teaching, Service and Community Practices and the results achieved at the FHU. Activities were monitored: home visits, lectures in the waiting room, Integrative and Complementary Practices, health fair, and matrix support; complementing the study, the Self-Reporting Questionnaire (SRQ-20) tool was used and the training of health professionals for later use of the tool in the community was used. Results: in the context of the students, the bond with the community made it possible to know the daily lives of patients, in its biopsychosocial scope, and contributed to humanized medical training. Professionals showed interest in improving the services offered, although there was resistance to operationalize activities in the unit's routine. The population that participated in the proposed activities rated them positively, despite the low adherence, limiting the scope of interventions. Conclusion: the lived experience proved to be beneficial for the consolidation of a new form of mental health care for patients at the FHU, consisting of teaching-service-community integration.
\end{abstract}

Keywords: Mental health; Home visit; Complementary therapies; Medical education; Primary health care.

\title{
Resumen
}

Objetivo: explicar las experiencias de estudiantes de segundo año de medicina sobre la implementación del proyecto de intervención en salud mental en una Unidad de Salud de la Familia (USF) en la ciudad de Jequié, Bahía, Brasil. Metodología: se trata de un relato de experiencia, que muestra la construcción del proyecto de intervención de la disciplina Integración Docente, Servicio y Prácticas Comunitarias y los resultados alcanzados en la USF. Se monitorearon las actividades: visitas domiciliarias, charlas en sala de espera, Prácticas Integrativas y Complementarias, feria de salud y apoyo matricial; como complemento del estudio, se utilizó la herramienta Cuestionario de Autoinforme (SRQ-20), así como la formación de los profesionales de la salud para su posterior uso en la comunidad. Resultados: en el contexto de los estudiantes, el vínculo con la comunidad permitió conocer la vida cotidiana de los pacientes, en su ámbito biopsicosocial, y contribuyó a la formación médica humanizada. Los profesionales mostraron interés en mejorar los servicios ofrecidos, aunque hubo resistencia a operativizar actividades en la rutina de la unidad. La población que participó en las actividades propuestas las calificó positivamente, a pesar de la baja adherencia, limitando el alcance de las intervenciones. Conclusión: la experiencia vivida resultó beneficiosa para la consolidación de una nueva forma de atención en salud mental para los pacientes de la USF, consistente en la integración enseñanza-servicio-comunidad.

Palabras clave: Salud mental; Visita domiciliaria; Terapias complementarias; Educación médica; Atención primaria de salud.

\section{Introdução}

As Práticas de Integração Ensino-Serviço-Comunidade (PIESC), um dos pilares da metodologia ativa adotada por alguns cursos de medicina, visam estabelecer o contato precoce entre o estudante e o Sistema Único de Saúde (SUS), por meio de atividades semanais e trabalho em equipe multiprofissional, que estimulam o desenvolvimento do olhar crítico perante a situação local de saúde e possibilitam o estabelecimento do vínculo com a equipe e a comunidade (Brandão \&, 2013). Dentro dessa perspectiva, estratégias de saúde são realizadas dentro das Unidades de Saúde da Família (USF) por meio de intervenções, voltadas à prevenção de agravos e à sensibilização da população acerca do tema escolhido.

Nesse contexto de Atenção Primária à Saúde (APS), criada com o objetivo de organizar o atendimento e distribuir os pacientes de forma mais efetiva, a Estratégia Saúde da Família (ESF) é um dos pilares da APS e representa a porta de entrada do SUS (Oliveira \& Pereira, 2013). Dentre todas as causas de procura do sistema, as psiquiátricas estão entre as principais, o que reforça a importância de discussão do tema em todos os níveis de atenção à saúde (Campos, et al., 2011). De acordo com dados do DATASUS (2021), durante o ano de 2018 e 2019, houve 3.233 notificações sobre violência interpessoal/autoprovocada, que estão incluídos os comportamentos suicidas e a autoagressão; nesses mesmos anos, a cidade 
de Jequié recebeu 61 notificações. As informações colhidas confirmam a necessidade de intervenções em saúde mental na comunidade.

A Organização Mundial da Saúde (OMS) tem destacado a importância das questões envolvendo a saúde mental desde a década de 1970, indicando a inserção de serviços de psiquiatria na atenção básica e a ampliação da participação comunitária (Oliveira, et al., 2017). Ainda assim, percebe-se como um dos principais desafios a serem enfrentados pelo SUS, a melhoria no acesso e na qualidade da atenção em saúde mental em uma Rede de Atenção Psicossocial, a fim de garantir a todo cidadão serviços de saúde inclusivos, integrais e de qualidade (Ministério da Saúde, 2015).

Outrossim, a alta prevalência dos transtornos mentais comuns, fenômenos que envolvem sintomas somáticos, ansiosos e depressivos, com repercussões nos âmbitos biológico, cultural, social, econômico e político, justificam a relevância das atividades das PIESC voltadas à saúde mental na APS. As visitas domiciliares que são uma das atividades padrões da disciplina, permitem o contato dos profissionais com a realidade dos indivíduos assistidos e o reconhecimento dos problemas e necessidades de saúde da comunidade, além de propiciar o estabelecimento do vínculo entre eles (Eufrásio \&, 2010; Assis \& Castro-Silva, 2018). Tal relação proporciona, dentre outros benefícios, a melhor adesão do indivíduo ao tratamento e o desenvolvimento de intervenções com maior potencial terapêutico (Ministério da Saúde, 2012; Chuengue \& Franco, 2018).

Nesse contexto, a ESF pode ser vista como um campo de práticas para a consolidação da integralidade, do rastreamento e da produção de novos modos de cuidado em saúde mental (Aosani \& Nunes, 2013; Lucchese \&, 2014; Murcho \&, 2016). Práticas como salas de espera dentro do espaço das unidades, podem potencializar discussões acerca do cotidiano das pessoas, proporcionando uma reflexão e posicionamento crítico para a qualidade de vida e manutenção da saúde, com a finalidade de amenizar o desgaste físico e emocional associado ao tempo de espera por uma consulta ou outro procedimento em saúde (Wild, et al., 2014).

As ações de educação em saúde pressupõem uma abordagem organizada e crítica, podendo atender às necessidades de atenção à saúde para a mudança e a autonomia dos usuários, fortalecendo a comunicação com o uso de uma linguagem concisa, clara e coerente, para que as pessoas possam compreender e refletir o que está sendo emitido. Com base nisso, as intervenções realizadas tiveram temas específicos de prevenção e educação, sempre trazendo a relação à saúde mental (Rosa \&, 2011). Também foram utilizadas como estratégias as Práticas Integrativas e Complementares (PIC) que visa garantir a integralidade na atenção à saúde. A partir de uma visão holística, o exercício dessas práticas leva em conta a singularidade do sujeito, estimula o autocuidado, valoriza a escuta acolhedora e desenvolve o vínculo terapêutico (Ministério da Saúde, 2015; Carvalho \& Nóbrega, 2017). Destacam-se, ademais, a musicoterapia e a meditação, que trazem como benefícios a diminuição dos níveis de ansiedade, a modificação positiva no humor, o equilíbrio emocional e a modulação da dor, sendo este último benefício também obtido pela aromaterapia, a partir da indução de secreção de $\beta$-endorfina (Melo, et al., 2018; Mascarenhas, et al., 2019). Por fim, a massoterapia tem efeitos de redução da ansiedade, frequência cardíaca e depressão, em resposta ao relaxamento alcançado (Kurebayashi, et al., 2016).

No contexto da atenção básica, o Self-Reporting Questionnaire (SRQ-20) constitui-se de uma ferramenta importante para rastreamento de transtornos mentais comuns, visto que é funcional em unidades de serviço de saúde onde há limitação de recursos financeiros e estruturais, havendo a necessidade unicamente de profissionais treinados para a sua aplicação (Van der Westhuizen \&, 2016). Associada a essas características inerentes ao instrumento, havia uma demanda, por parte dos agentes comunitários de saúde (ACS) da USF em questão, para o rastreamento de agravos à saúde mental, uma vez que a suspeita desses transtornos frequentemente se dava por parâmetros não sistematizados. Por fim, o Apoio Matricial organiza-se numa metodologia de trabalho para além daquela preconizada pelo sistema hierarquizado, enrijecido em mecanismos de referência e contrarreferência, protocolos e centros de regulação (Gonçalves, et al., 2011). 
Com base nesses pressupostos, o estudo objetiva explanar as vivências de graduandos do segundo ano de medicina, relativas à implantação do projeto de intervenção em saúde mental em uma USF no município de Jequié, Bahia, Brasil.

\section{Metodologia}

As intervenções em saúde mental foram realizadas pelos discentes do curso de medicina, juntamente aos ACS, com os pacientes adscritos na equipe dois de uma USF do município de Jequié, que está localizado na região sudoeste da Bahia com população estimada em 151.895 no último censo em 2010. Na cidade, há 85 estabelecimentos de saúde do SUS, dentre as quais, 21 são USF (IBGE, 2019; Ministério da Saúde, 2019). As ações de saúde são atividades obrigatórias da disciplina PIESC e o tema é escolhido a partir da observação dos principais problemas evidenciados pelos pacientes. Por sua vez, a USF é escolhida pela universidade e pela Secretaria de Saúde do município.

As atividades ocorreram no período de outubro de 2018 a agosto de 2019 e como estratégias metodológicas foram utilizadas visitas domiciliares, palestras em sala de espera, PIC, como a musicoterapia, meditação guiada, aromaterapia e massoterapia, feira de saúde e instrução acerca do matriciamento entre estabelecimentos de saúde. A primeira sala de espera teve como assunto a campanha Novembro Azul, cuja abordagem correlacionou o câncer de próstata e os impactos causados à saúde mental do acometido e dos seus familiares. Em outros encontros, discutiu-se sobre ansiedade, depressão e prevenção contra o suicídio, os quais foram ministrados por especialistas. As atividades realizadas tiveram cunho interativo para que os usuários presentes, bem como os profissionais da USF, pudessem expressar dúvidas e opiniões, acrescentando conhecimento às discussões.

Além disso, foi realizada capacitação dos ACS para a utilização da versão brasileira do SRQ-20, instrumento de rastreamento de transtornos mentais comuns recomendado pela OMS para estudos comunitários e em atenção básica à saúde, que contém uma escala dicotômica (sim/não) para cada um dos seus 20 itens, divididos de acordo com os seguintes grupos de sintomas: humor depressivo/ansioso, sintomas somáticos, decréscimo de energia vital e pensamentos depressivos (Gonçalves \&, 2008; Carvalho \&, 2016).

A partir da identificação da necessidade de comunicação entre a USF e os outros níveis de complexidade, realizou-se um encontro com os profissionais envolvidos, com o objetivo de sensibilizá-los a respeito da importância do matriciamento. No encontro, foi entregue aos profissionais um fluxograma informativo acerca dos estabelecimentos de saúde que realizam atendimentos voltados aos agravos à saúde mental.

A última intervenção adotada pelo grupo, visando à manutenção do vínculo e o bem-estar da população, foi a realização de uma Feira de Saúde. Estandes foram instalados para oferecer ao público diferentes atividades, tais como: PIC, teste de glicemia e aferição da pressão arterial, medidas antropométricas (índice de massa corpórea, relação cintura-quadril e percentual de gordura), testes rápidos, orientação nutricional, informações acerca do funcionamento dos Centros de Atenção Psicossocial e palestra sobre Saúde Mental. Ao final de todas ações realizadas semanalmente, os discentes utilizaram a observação sistemática como estratégia de acompanhamento e avaliação das atividades.

Não foi preciso submeter o trabalho ao Comitê de Ética e Pesquisa, visto que o presente estudo se trata de um relato de experiência e não revela dados que possam identificar os sujeitos.

\section{Resultados e Discussão}

A experiência vivenciada mostrou-se benéfica para a consolidação de uma nova forma de cuidado na USF que consiste na integração ensino-serviço-comunidade. No contexto dos discentes, o vínculo com a comunidade possibilitou o conhecimento do cotidiano dos pacientes, em seu âmbito biopsicossocial, e contribuiu para a formação médica humanizada. No que tange aos profissionais, percebeu-se o interesse na melhoria dos serviços oferecidos, embora houvesse uma resistência 
na operacionalização das atividades na rotina da unidade. Por sua vez, a população que aguardava atendimento na USF constituiu-se como o principal público das ações desenvolvidas na unidade e as avaliou positivamente. Destaca-se, no entanto, como aspecto negativo, a baixa adesão da maioria da população acompanhada nas visitas domiciliares às atividades propostas, limitando o alcance das intervenções.

Em relação às visitas domiciliares, tal vínculo constituiu uma ferramenta importante para atingir os objetivos propostos, pois a comunicação efetiva criou um ambiente propício para o compartilhamento de vivências, o que permitiu a abordagem do tema proposto pelos discentes. Todo o planejamento das visitas foi feito a partir da observação do modo de vida da comunidade, seus recursos, conflitos e valores culturais, resultando em uma melhor assistência multiprofissional aos indivíduos (Nascimento \&, 2013).

As salas de espera mostraram-se eficazes para todos os envolvidos, seja na aprendizagem de novos conhecimentos, no compartilhamento de vivências e saberes, na identificação de temas que a comunidade considera interessante e pertinente e na criação de vínculo entre profissionais, usuários e estudantes (Feitosa, et al., 2019).

As PIC foram as atividades com melhor retorno pela comunidade. A musicoterapia, meditação, aromaterapia e massoterapia se mostraram eficazes na intervenção da saúde mental dos usuários. As PIC se mostraram instrumentos de escuta acolhedora, vínculo terapêutico, autocuidado e integração do indivíduo com a comunidade e a unidade de saúde (Carvalho \& Nóbrega, 2018). A feira de saúde foi um evento considerado de valor educativo, visto que informou e sensibilizou a comunidade no que concerne à melhoria da qualidade de vida a partir da prevenção, orientando para a mudança de hábitos de vida.

O matriciamento se mostrou essencial para o contato entre a unidade de saúde e centros especializados em saúde mental na cidade, tendo em vista que o encaminhamento deve ser realizado para diferentes níveis de atenção de acordo com a classificação de gravidade do paciente, o que não era observado na USF de estudo. Assim sendo, o matriciamento em saúde mental facilita o direcionamento dos fluxos na rede, promovendo o vínculo entre as diversas unidades que trabalham com a saúde mental da população (Gazignato \& Silva, 2014).

O uso do SRQ-20 mostrou-se eficaz para a suspeição de transtornos mentais e permitiu uma visão mais ampliada dos problemas de saúde da comunidade. Assim, a capacidade de utilização do SRQ pelos ACS, que se constituem como principais formadoras do vínculo com a comunidade, permite que o rastreamento de indivíduos com possíveis transtornos mentais comuns, ocorra da forma mais precoce possível, possibilitando uma referenciação mais efetiva (Santos \&, 2010).

Dessa forma, torna-se imprescindível o engajamento dos profissionais e uma maior interação com outras USF que já incluíram na sua rotina atividades que estavam relacionadas à saúde mental. Em relação à baixa adesão é viável a execução de mais estratégias em outros ambientes públicos e privados, como as igrejas e as escolas, com a finalidade de motivar a continuação das ações de saúde nessa temática.

\section{Conclusão}

A participação de estudantes, profissionais de diversas áreas e usuários da unidade possibilitou a aproximação entre as partes, a partir de um trabalho coletivo de compartilhamento de saberes, construindo teorias, conceitos e abordagens para abranger a complexidade que envolve as discussões sobre saúde mental no nível primário de atendimento à comunidade. Por fim, a experiência vivenciada mostrou-se benéfica para a consolidação de uma nova forma de cuidado à saúde mental dos pacientes da USF, consistindo na integração ensino-serviço-comunidade.

\section{Referências}

Aosani, T. R., \& Nunes, K. G. (2013). A saúde mental na atenção básica: a percepção dos profissionais de saúde. Revista Psicologia e Saúde, 5(2). 
Araújo, P. C. (2013). Trabalho em equipe multiprofissional na Estratégia de Saúde da Família: a interface entre a equipe de Saúde Bucal e a equipe de Saúde da Família (Doctoral dissertation).

Assis, A. S. D., \& Castro-Silva, C. R. D. (2018). Agente comunitário de saúde e o idoso: visita domiciliar e práticas de cuidado. Physis: Revista de Saúde Coletiva, 28, e280308.

Brandão, E. R. M., Rocha, S. V., \& Silva, S. S. D. (2013). Práticas de integração ensino-serviço-comunidade: reorientando a formação médica. Revista Brasileira de Educação Médica, 37, 573-577.

Caires, J. S., Andrade, T. A., do Amaral, J. B., de Andrade Calasans, M. T., \& da Silva Rocha, M. D. (2014). A utilização das terapias complementares nos cuidados paliativos: benefícios e finalidades. Cogitare enfermagem, 19(3).

Campos, R. O., Gama, C. A., Ferrer, A. L., Santos, D. V. D. D., Stefanello, S., Trapé, T. L., \& Porto, K. (2011). Saúde mental na atenção primária à saúde: estudo avaliativo em uma grande cidade brasileira. Ciência \& Saúde Coletiva, 16, 4643-4652.

Carvalho, D. B. D., Araújo, T. M. D., \& Bernardes, K. O. (2016). Transtornos mentais comuns em trabalhadores da Atenção Básica à Saúde. Revista Brasileira de Saúde Ocupacional, 41.

Carvalho, J. L. D. S., \& Nóbrega, M. D. P. S. D. S. (2018). Práticas integrativas e complementares como recurso de saúde mental na Atenção Básica. Revista Gaúcha de Enfermagem, 38.

Chiaverini, D. H., Gonçalves, D. A., Ballester, D., Tófoli, L. F., Chazan, L. F., Almeida, N., \& Fortes, S. (2011). Guia prático de matriciamento em saúde mental.

Chuengue, A. P. G., \& Franco, T. B. (2019). O reconhecer e o lidar dos agentes comunitários de saúde diante da bioética: entre a ética do cuidado e os poderes disciplinares. Physis: Revista de Saúde Coletiva, 28.

Eufrásio, R. G., de Alcântara Junior, J. E., Araújo, L. R., \& de Negreiros, M. A. M. P. (2010). Detecção Precoce De Carcinoma Basocelular: Importância De Visita Domiciliar No Contexto Integral Do Idoso. Revista de APS, 13(2).

Feitosa, A. L. F., Silva, R. L., Santos, K. S. O., Silva, L. K. G., Rocha, M. C. G., \& Andrade, M. F. L. O. (2019). Sala de espera: estratégia de educação em saúde no contexto da atenção básica. Revista Brasileira de Educação e Saúde, 9(2), 67-70.

Ferreira, M. L. S., Moura, J. F. L., Silva, E. S., Rocha, R. F., Olivares, A. I. O., \& Hayd, R. L. N. (2010). Feira de saúde do curso de medicina da UFRR: uma aproximação com a comunidade. Revista Brasileira de Educação Médica, 34, 310-314.

Gazignato, E. C. D. S., \& Silva, C. R. D. C. (2014). Saúde mental na atenção básica: o trabalho em rede e o matriciamento em saúde mental na Estratégia de Saúde da Família. Saúde em Debate, 38, 296-304.

Gonçalves, D. M., Stein, A. T., \& Kapczinski, F. (2008). Avaliação de desempenho do Self-Reporting Questionnaire como instrumento de rastreamento psiquiátrico: um estudo comparativo com o Structured Clinical Interview for DSM-IV-TR. Cadernos de saúde pública, 24, 380-390.

Instituto Brasileiro de Geografia e Estatística. (n.d.). IBGE Cidades - Jequié/BA. https://cidades.ibge.gov.br/brasil/ba/jequie/panorama.

Kurebayashi, L. F. S., Turrini, R. N. T., Souza, T. P. B. D., Takiguchi, R. S., Kuba, G., \& Nagumo, M. T. (2016). Massage and Reiki used to reduce stress and anxiety: Randomized Clinical Trial. Revista latino-americana de enfermagem, 24.

Lima, C. D. A., Santos, A. M. V. D. S., Messias, R. B., Costa, F. M. D., Barbosa, D. A., Silva, C. S. D. O., \& Brito, M. F. S. F. (2018). Práticas integrativas e complementares: utilização por agentes comunitários de saúde no autocuidado. Revista Brasileira de Enfermagem, 71, 2682-2688.

Lucchese, R., Sousa, K. D., Bonfin, S. D. P., Vera, I., \& Santana, F. R. (2014). Prevalência de transtorno mental comum na atenção primária. Acta Paulista de Enfermagem, 27, 200-207.

Mascarenhas, V. H. A., Lima, T. R., Silva, F. M. D., Negreiros, F. D. S., Santos, J. D. M., Moura, M. Á. P., \& Jorge, H. M. F. (2019). Evidências científicas sobre métodos não farmacológicos para alívio a dor do parto. Acta Paulista de Enfermagem, 32, 350-357.

Melo, G. A. A., Rodrigues, A. B., Firmeza, M. A., Grangeiro, A. S. D. M., Oliveira, P. P. D., \& Caetano, J. Á. (2018). Musical intervention on anxiety and vital parameters of chronic renal patients: a randomized clinical trial. Revista latino-americana de enfermagem, 26.

Ministério da Saúde. (2012). Política Nacional de Atenção Básica. http://189.28.128.100/dab/docs/publicacoes/geral/pnab.pdf.

Ministério da Saúde. (2015). Caderno HumanizaSUS (5th ed.). http://bvsms.saude.gov.br/bvs/publicacoes/saude_mental_volume_5.pdf.

Ministério da Saúde. (2015). Política nacional de práticas integrativas e complementares no SUS: atitude de ampliação de acesso. https://bvsms.saude.gov.br/bvs/publicacoes/politica_nacional_praticas_integrativas_complem entares_2ed.pdf. 12

Ministério da Saúde. Departamento de Informática do SUS. Cadastro Nacional de Estabelecimentos de Saúde. http://cnes2.datasus.gov.br/Listar_Mantidas.asp?VCnpj=13894878000160\&VEstado=29\&VN ome=PREFEITURA\%20MUNICIPAL\%20DE\%20JEQUIE. 11

Ministério da Saúde. Sistema de Informação de Agravos de Notificação (2021, March 18). Violência Interpessoal/Autoprovocada. DATASUS. http://tabnet.datasus.gov.br/cgi/tabcgi.exe?sinannet/cnv/violebr.def

Murcho, N., Pacheco, E., \& Jesus, S. (2016). Transtornos mentais comuns nos Cuidados de Saúde Primários: Um estudo de revisão. Revista portuguesa de enfermagem de saúde mental, 15, 30-36. 
Research, Society and Development, v. 10, n. 13, e215101321090, 2021

(CC BY 4.0) | ISSN 2525-3409 | DOI: http://dx.doi.org/10.33448/rsd-v10i13.2109으

Nascimento, J. S., Costa, L. D. M. C., dos Santos, R. M., \& dos Anjos, D. S. (2013). Visitas domiciliares como estratégias de promoção da saúde pela enfermagem. Revista Brasileira em Promoção da Saúde, 26(4), 513-522.

Oliveira, E. C. D., Medeiros, A. T. D., Trajano, F. M. P., Chaves, G., Almeida, S. A. D., \& Almeida, L. R. D. (2017). O cuidado em saúde mental no território: concepções de profissionais da atenção básica. Escola Anna Nery, 21.

Oliveira, M. A. D. C., \& Pereira, I. C. (2013). Atributos essenciais da atenção primária e a estratégia saúde da família. Revista Brasileira de Enfermagem, 66, 158-164.

Rosa, J., Barth, P. O., \& Germani, A. R. M. (2011). A sala de espera no agir em saúde: espaço de educação e promoção à saúde. Perspectiva, Erechim, 35(129), 121-130.

Santos, K. O. B., de Araújo, T. M., de Sousa Pinho, P., \& Silva, A. C. C. (2010). Avaliação de um instrumento de mensuração de morbidade psíquica: estudo de validação do Self-Reporting Questionnaire (SRQ-20). Revista Baiana de Saúde Pública, 34(3), 544-544.

Van der Westhuizen, C., Wyatt, G., Williams, J. K., Stein, D. J., \& Sorsdahl, K. (2016). Validation of the self reporting questionnaire 20-item (SRQ-20) for use in a low-and middle-income country emergency centre setting. International journal of mental health and addiction, 14(1), 37-48.

Wild, C. F., da Silveira, A., Favero, N. B., de Oliveria Rosa, E., Gueterres, É. C., \& de Souza Leal, S. D. (2014). Educação em saúde na sala de espera de uma policlínica infantil: relato de experiência. Revista de Enfermagem da UFSM, 4(3), 660-666. 